\title{
Cytokine and angiogenic factors associated with efficacy and toxicity of pazopanib in advanced soft-tissue sarcoma: an EORTC-STBSG study
}

\section{S Sleijfer ${ }^{*, 1}$, T Gorlia ${ }^{2}$, C Lamers', H Burger', J-Y Blay ${ }^{3}$, A Le Cesne ${ }^{4}$, M Scurr ${ }^{5}$, F Collin ${ }^{6}$, L Pandite ${ }^{7}$, $S$ Marreaud ${ }^{2}$ and $P$ Hohenberger ${ }^{8}$}

'Department of Medical Oncology, Erasmus University Medical Center, Daniel den Hoed Cancer Center, Groene Hilledijk 30 I, 3075 EA Rotterdam, The Netherlands; ' $E$ ERTC Headquarters, Brussels, Belgium; ${ }^{3}$ Department of Medical Oncology, Centre Leon Berard, Lyon, France; ${ }^{4}$ Department of Medical Oncology, Institute Gustave Roussy, Villejuif, France; ${ }^{5}$ Sarcoma Unit, Royal Marsden Hospital, London, UK; ${ }^{6}$ Department of Pathology, Center Georges-François-Leclerc, Dijon, France; 7 GlaxoSmithKline, Research Triangle Park, North Carolina, USA; ${ }^{8}$ Department of Surgery, University of Heidelberg, Mannheim, Germany

BACKGROUND: Pazopanib has activity in relapsed non-adipocytic soft-tissue sarcomas (STS). A series of serum cytokines and angiogenic factors (CAFs) at baseline and changes in soluble vascular endothelial growth factor receptor-2 (sVEGFR2) or placental-derived growth factor (PIGF) levels during treatment were explored for their association with outcome.

METHODS: Twenty-three baseline CAFs, and sVEGFR2 and PIGF changes were measured in 85 and 32 patients, respectively. Associations between baseline CAF levels and efficacy parameters, plus between-week 12 sVEGFR2 and PIGF levels and pazopanibspecific toxicities were investigated.

RESULTS: At baseline, low interleukin (IL)- 12 p40 subunit and MPC3 levels were associated with better progression-free survival (PFS) at 12 weeks (PFS 12 wks), low basic nerve growth factor and hepatocyte growth factor with a better PFS, and low inter-cellular adhesion molecule-I and IL-2 receptor alpha with prolonged overall survival (OS; all $P<0.05)$. Pazopanib decreased sVEGFR2 and increased PIGF levels. Low SVEGFR2 and high PIGF levels at week 12 were associated with higher-grade hypertension, with TSH elevations and with poorer PFS 12 wks, and OS (both $P<0.05$ ).

CONCLUSION: Several baseline CAFs were related to outcome parameters. Low sVEGFR2 and high PIGF at week 12 associate with several pazopanib-specific toxicities and poorer efficacy. If confirmed, these factors may be used as early markers for response to and toxicity from pazopanib, enabling further individualisation of STS treatment.

British Journal of Cancer (2012) 107, 639-645. doi:I0.1038/bjc.2012.328 www.bjcancer.com

Published online 17 July 2012

(C) 2012 Cancer Research UK

Keywords: soft-tissue sarcomas; pazopanib; cytokines; prognosis

The group of adult soft-tissue sarcomas (STS) is a rare group of tumours comprising more than 40 entities. Patients with advanced STS have a poor prognosis, and until recently, only doxorubicin and ifosfamide had shown consistent activity (Sleijfer et al, 2005; Tascilar et al, 2007). Fortunately, with an increasing recognition that these histological subtypes have diverse natural histories and treatment responses, an increasing number of new systemic treatments based on histology are now being utilised, including imatinib for gastro-intestinal stromal tumours (GISTs; Verweij et al, 2004), taxanes for angiosarcomas (Fata et al, 1999), and trabectedin for liposarcomas and leiomyosarcomas (Demetri et al, 2009). Undoubtedly though, the vast majority of advanced non-GISTs STS patients continue to face a poor prognosis. The median overall survival (OS) for patients with metastatic STS does not exceed 12 months and this statistic has not changed in more than two decades, clearly underscoring the need for effective novel agents.

*Correspondence: Dr S Sleijfer; E-mail: s.sleijfer@erasmusmc.nl Received 10 February 2012; revised 19 June 2012; accepted 22 June 2012; published online 17 July 2012
Recently, the European Organization of Research and Treatment of Cancer, Soft Tissue, and Bone Sarcoma Group (EORTC-STBSG) performed a phase II study of pazopanib in patients with relapsed or refractory STS (Sleijfer et al, 2009). Pazopanib is a tyrosinekinase inhibitor (TKI) targeting several factors, among which are the vascular endothelial growth factor receptors (VEGFRs), the platelet-derived growth factor receptors, and the c-kit. Stratification for histological subtype was undertaken and clinically significant anti-tumour activity was seen in those patients with synovial sarcomas, leiomyosarcomas, and other eligible STS entities, but not for those with adipocytic sarcomas (Sleijfer et al, 2009). On the basis of this study, a placebo-controlled phase III study in non-adipocytic STS was initiated by the EORTCSTBSG group and showed a three-fold prolongation in progression-free survival (PFS; Van der Graaf et al, 2011).

The role of biomarkers for either toxicity or efficacy has become increasingly important in allowing 'personalisation' of particular treatment types for many cancers. As with other VEGFR-TKIs, pazopanib's main mechanism of action is thought to be through impacting tumour angiogenesis (Hamberg et al, 2010). Therefore, as part of the phase II study of pazopanib in advanced STS, 
an exploratory translation research component was incorporated to attempt to identify cytokines and angiogenic factors (CAFs) determined at baseline that are associated with outcome to pazopanib in STS. In addition, VEGFR-TKIs have been shown to decrease soluble VEGFR2 (sVEGFR2) and increase placentalderived growth factor (PlGF) blood levels (Rini et al, 2008; Hanrahan et al, 2009a; Bass et al, 2010; Murukesh et al, 2010; Nikolanikos et al, 2010), and consequently, these markers have been suggested as pharmacodynamic markers for VEGFR inhibition. We therefore also analysed whether changes in PlGF and sVEGFR2 correlate with the occurrence of toxicities thought to be specific for pazopanib and with survival outcome.

\section{METHODS}

\section{Patients and study design}

The details of the multi-centre, single-arm phase II study of pazopanib in relapsed or refractory STS (EORTC 62043, NCT000 297258) have been previously published (Sleijfer et al, 2009). Briefly, patients with intermediate or high-grade advanced STS, ineligible for further chemotherapy, or having received no more than two prior cytotoxic agents in the advanced setting were eligible. Patients had documented progression before study entry, adequate performance status, and a good organ function. Pazopanib was given a dose of $800 \mathrm{mg}$ daily until progression or unacceptable toxicity. The primary end point was progression-free rate at 12 weeks. Secondary end points included response, safety, and OS. Given the great heterogeneity of the different STS entities, four different strata were studied: adipocytic STS, leiomyosarcomas, synovial sarcomas, and other eligible STS types. A Simon two-stage design was applied (P1: $40 \% ; \mathrm{P} 0: 20 \% ; \alpha=\beta=0.1$ ) to each stratum. In total, 142 patients were enroled. The adipocytic STS stratum was closed after the first stage, given insufficient activity according to study protocol, whereas in the other three cohorts, pazopanib yielded anti-tumour activity considered worthwhile to be further explored according to the pre-specified criteria (Sleijfer et al, 2009). The study was approved by the institutional review boards of all participating centres and was conducted in agreement with the Declaration of Helsinki and the International Conference on Harmonization of Good Clinical Practice guidelines. All patients gave written informed consent to participate. Consent for blood sampling for biomarker analysis was optional. There was a separate consent for participation in the translational research part of the study and this part of the study was voluntary.

\section{Blood sample collection and analyses}

Venous serum samples were taken at baseline and at 12 weeks after treatment start, and stored at $-20^{\circ} \mathrm{C}$ until analysis. Analysis of serum samples was performed blinded to patient outcome. Twenty-three different CAFs were determined at baseline using a commercially available multiplex bead assay (BioRab Laboratories, Inc., Veenendaal, The Netherlands/Minneapolis, MN, USA) according to the manufacturers' instructions. In addition, PlGF and sVEGFR2 were measured at baseline and at the time of evaluation of the primary end point (12 weeks after treatment start) by enzyme-linked immunosorbent assay (DPG00 and DVR200, respectively) according to the manufacturers' instruction (R\&D systems Europe, Abingdon, UK).

\section{Statistical methods}

The aim of this translational research sub-study was to generate hypotheses that could be further validated in future trials with pazopanib in STS and so, these analyses are essentially exploratory.
With respect to the CAF determined at baseline, we aimed to identify biomarkers that could predict 'success of therapy', defined as being PFS at 12 weeks $\left(\mathrm{PFS}_{12 \mathrm{wks}}\right)$, which was the primary end point of the clinical trial. Secondary objectives included correlation with other efficacy end points, PFS, and OS. The CAF analytes were first analysed as continuous variables; measurements below the detection level were conventionally assigned a value equal to half of the detection threshold. For illustration of the results, significant factors were subsequently categorised according to quartiles (rounded values).

In addition to baseline, sVEGFR2 and PIGF serum levels were determined at 12 weeks after treatment start. We analysed whether the specific pazopanib toxicities hypertension and hypopigmentation could be predicted by absolute levels and/or changes in these two factors. Secondary objectives included correlation with other toxicities (fatigue, diarrhoea, nausea, and TSH elevation), and with the efficacy end points PFS $_{12 \mathrm{wks}}$, PFS, and OS. Analyses were done for the serum level at baseline, the serum level at 12 weeks, and the ratio between the two levels. All these three determinants were initially explored as continuous variables and subsequently categorised as binary variables (using the median value as cut-off) for illustration of the results.

The categorical data mentioned in this report were summarised by frequencies and percentages, the continuous covariates by median, range, and numbers of observations. Survival data were plotted using the Kaplan-Meier method. Toxicity was graded according to the NCI-CTCAE version 3.0 (http://ctep.cancer.gov).

Univariate analysis was performed among baseline covariates (CAFs) by logistic regression (Wald test) for $\mathrm{PFS}_{12 \mathrm{wks}}$, and by Cox regression (Wald test) for PFS and OS, when analysed as continuous variables. The statistical significance was set at 0.05 based on a two-sided statistical test. Subsequently, multivariate regression models were built; a logistic model for $\mathrm{PFS}_{12 \mathrm{wks}}$ and Cox models for OS and PFS including all factors with a significant or borderline prognostic value in the univariate analyses. Nonsignificant factors were removed according to a backward selection procedure with the statistical significance set at 0.05 based on a two-sided test. The univariate analysis was the first step of the process aiming at rejecting the less significant variables from the multivariate regression models.

Outcomes are reported as odds ratios (OR; $\left.\mathrm{PFS}_{12 \mathrm{wks}}\right)$ or hazard ratios (HR; PFS and OS) with confidence intervals (CIs).

Correlations between baseline CAFs and $\mathrm{PFS}_{12 \mathrm{wks}}$ were subsequently illustrated in bar charts, and correlations with PFS and OS in Kaplan-Meier curves; for this purpose, the baseline variables were categorised.

Pearson's and Spearman's correlation coefficients were used to assess the relationship between sVEGFR2 and PIGF levels (continuous variables) and toxicities. Results were illustrated as bar charts, using binary variables.

Statistical Analysis Software version 9.2 (SAS Institute, Cary, NC, USA) was used for statistical analyses.

\section{RESULTS}

\section{Patient population}

A total of 142 patients were recruited in the clinical trial from 15 November 2005 to 16 May 2007. The CAF profile (at baseline) could be measured in 85 of those patients. The sVEGFR 2 and PlGF levels were measured in 32 and 31 patients, respectively, at baseline and at 12 weeks after treatment initiation. There were no significant differences between the characteristics of the 142 patients comprising the original study population compared with those incorporated in this side-study (Table 1). 
Table I Patients' and disease characteristics

\begin{tabular}{|c|c|c|c|}
\hline & $\begin{array}{l}\text { All patients } \\
(N=142)\end{array}$ & $\begin{array}{c}\text { CAF study } \\
(N=85)\end{array}$ & $\begin{array}{l}\text { sVEGFR2/PIGF } \\
\text { study }(N=32)\end{array}$ \\
\hline \multirow{2}{*}{\multicolumn{4}{|c|}{ Age (years) }} \\
\hline & & & \\
\hline Median & 51.4 & 51.7 & 46.0 \\
\hline Range & | $8.5-79.3$ & 18.5-77.3 & | $8.5-73.4$ \\
\hline \multicolumn{4}{|l|}{ Sex } \\
\hline Male & $7 \mid(50.0)$ & $43(50.6)$ & $14(43.8)$ \\
\hline Female & $71(50.0)$ & $42(49.4)$ & $18(56.3)$ \\
\hline \multicolumn{4}{|l|}{ Performance status (WHO) } \\
\hline 0 & $72(50.7)$ & $42(49.4)$ & $20(62.5)$ \\
\hline । & $70(49.3)$ & $43(50.6)$ & $12(37.5)$ \\
\hline \multicolumn{4}{|l|}{ Time since diagnosis (d) } \\
\hline Median & 872.5 & 895.0 & 878.5 \\
\hline Range & $90.0-10481.0$ & $90.0-7488.0$ & | $89.0-7488.0$ \\
\hline \multicolumn{4}{|l|}{ Tumour grade } \\
\hline Low & $7(4.9)$ & $3(3.5)$ & $3(9.4)$ \\
\hline Intermediate & $67(47.2)$ & $42(49.4)$ & $13(40.6)$ \\
\hline High & $65(45.8)$ & $39(45.9)$ & $16(50.0)$ \\
\hline Unknown & $3(2.1)$ & I (I.2) & $0(0.0)$ \\
\hline \multicolumn{4}{|l|}{ Histology } \\
\hline Adipocytic (LIP) & $19(13.4)$ & $10(11.8)$ & $3(9.4)$ \\
\hline Fibroblastic & $5(3.5)$ & $4(4.7)$ & $2(6.3)$ \\
\hline Fibrohistiocytic (MFH) & $4(2.8)$ & $0(0.0)$ & $0(0.0)$ \\
\hline Smooth muscles (LMS) & $42(29.6)$ & $27(31.8)$ & $9(28.1)$ \\
\hline Skeletal muscle & ( 0.7$)$ & $1(1.2)$ & $0(0.0)$ \\
\hline Vascular & $5(3.5)$ & $4(4.7)$ & $1(3.1)$ \\
\hline Uncertain different. & $45(31.7)$ & $26(30.6)$ & $12(37.5)$ \\
\hline MPNST & $5(3.5)$ & $2(2.4)$ & $2(6.3)$ \\
\hline Malignant solitary fibrous & $3(2.1)$ & $2(2.4)$ & $2(6.3)$ \\
\hline Undifferentiated NOS & $11(7.7)$ & $8(9.4)$ & $1(3.1)$ \\
\hline GIST (ineligible) & I (0.7) & $1(1.2)$ & $0(0.0)$ \\
\hline Mesothelioma (ineligible) & I (0.7) & $0(0.0)$ & $0(0.0)$ \\
\hline
\end{tabular}

Abbreviations: $\mathrm{CAF}=$ cytokines and angiogenic factor; $\mathrm{GIST}=$ gastro-intestinal stromal tumour; LIP = liposarcoma; LMS = leiomyosarcoma; $\mathrm{MFH}=$ malignant fibrous histiocytoma; MPNST = malignant peripheral nerve sheath tumour; $N O S=$ not otherwise specified; PIGF = placental-derived growth factor, sVEGFR = soluble vascular endothelial growth factor receptor; $\mathrm{WHO}=$ World Health Organisation.

\section{Baseline CAF profile}

Table 2 depicts the distribution of the 23 markers measured at baseline. Univariate logistic regression models were built to explore the possible prognostic value of the different marker levels for $\mathrm{PFS}_{12 \mathrm{wks}}$. Only two factors, the interleukin (IL)-12 p40 subunit (IL12p40; OR 0.999; 95\% CI 0.998-1.000 $(P=0.0305)$ ) and monocyte chemotactic protein-3 (OR 0.978 ; 95\% CI $0.959-0.997$ $(P=0.0271))$, were significant at the 0.05 confidence level. Low levels of these factors were associated with a higher chance of being progression-free at 12 weeks. However, because of multiple testing, the false discovery rate adjusting for 23 tests was around $50 \%$. These two factors were highly correlated and both lost their significance in a multivariate model.

Two parameters were significantly correlated with prolonged PFS; low levels of hepatocyte growth factor (HGF; HR 1.000; 95\% CI $1.000-1.000(P=0.0079))$ and basic nerve growth factor (bNGF; HR 1.167; 95\% CI 1.049-1.299 $(P=0.0044)$ ). Again, because of multiple testing, the false discovery rate was relatively high at around 20\%. These two factors kept a significant prognostic value in the multivariate model. Results of these two correlations are shown as a Kaplan-Meier estimate of PFS in marker categories (Figure 1A and B).
Table 2 Distribution of the 23 CAF markers at baseline (all concentrations are in $\mathrm{pg} \mathrm{ml}^{-1}$ )

\begin{tabular}{|c|c|c|c|c|c|c|}
\hline Marker & $N$ & Minimum & Maximum & Median & $\begin{array}{l}\text { Detection } \\
\text { threshold }\end{array}$ & $\begin{array}{l}\mathrm{Nr}< \\
\text { thresh }\end{array}$ \\
\hline ILla & 85 & 2.5 & 15.2 & 2.5 & 5 & 79 \\
\hline IL2ra & 85 & 78 & 1258 & 344 & & \\
\hline IL3 & 85 & $50^{\mathrm{a}}$ & 613 & 188 & 100 & 28 \\
\hline ILI2p40 & 85 & 284 & 2524 & 1215 & & \\
\hline ILI6 & 85 & 61 & 2124 & 243 & & \\
\hline ILI8 & 85 & 45 & 724 & 110 & & \\
\hline CTACK & 85 & 80 & 3585 & 1280 & & \\
\hline GROa & 85 & 117 & 645 & 225 & & \\
\hline HGF & 85 & 551 & 15466 & 1330 & & \\
\hline ICAM-I & 85 & 21281 & 275798 & 82786 & & \\
\hline IFNa2 & 85 & 140 & 376 & 236 & & \\
\hline LIF & 85 & $10.0^{a}$ & 535 & 10.0 & 20 & 52 \\
\hline MCP3 & 85 & $4.5^{\mathrm{a}}$ & 105 & 48 & 9 & I \\
\hline MCSF & 85 & 40 & 1130 & 94 & & \\
\hline XMIF & 85 & 138 & 100000 & 2144 & & \\
\hline XMIG & 85 & 135 & 4451 & 701 & & \\
\hline bNGF & 85 & $2.0^{\mathrm{a}}$ & 9.5 & 2.0 & 4 & 50 \\
\hline SCF & 85 & $10.0^{\mathrm{a}}$ & 656 & 156 & 20 & 1 \\
\hline SCGFb & 85 & 2759 & 200000 & 53267 & & \\
\hline SDFla & 85 & 438 & 73250 & 981 & & \\
\hline TNFb & 85 & $4.0^{\mathrm{a}}$ & 103 & 19 & 8 & 14 \\
\hline TRAIL & 85 & 114 & 1100 & 520 & & \\
\hline VCAMI & 85 & 1464 & 280068 & 96161 & & \\
\hline
\end{tabular}

Abbreviations: $\mathrm{bNGF}=$ basic nerve growth factor; $\mathrm{CAF}=$ cytokines and angiogenic factor; HGF = hepatocyte growth factor; ICAM-I = inter-cellular adhesion molecule-I; IFNa2 =interferon alpha-2; ILIa =interleukin-I alpha; IL2ra $=\mathrm{IL}-2$ receptor alpha; ILI2p40=IL-12 p40 subunit; $M C P 3=$ monocyte chemotactic protein-3; $\mathrm{MCSF}=$ macrophage colony-stimulating factor; $\mathrm{TNFb}=$ tumour necrosis factor beta; TRAIL = TNF-related apoptosis-inducing ligand; VCAMI = vascular cell adhesion protein-I. ${ }^{a}$ Below the detection threshold.

Similarly, two baseline CAF levels demonstrated significant correlation with OS; low levels of IL-2 receptor alpha (IL2ra; HR 1.002; 95\% CIs 1.001-1.003 $(P=0.0078))$ and inter-cellular adhesion molecule-1 (ICAM-1; HR 1.000; 95\% CIs 1.000-1.000 $(P=0.0072))$ were associated with prolonged survival. Again, the false discovery rate adjusting for 23 tests was rather high around $20 \%$. Both factors were highly correlated and lost their significance in the multi-variate Cox model. Figure $1 \mathrm{C}$ and D depicts the OS in four markers categories.

\section{Placental-derived growth factor and sVEGFR2 at week 12}

The sVEGFR2 (32 patients) and PIGF (31 patients) serum levels were measured before treatment start and at the time of evaluation of the primary end point (end of week 12). It should be noted that those patients are not fully representative of the study population. Patients had to be alive and under protocol therapy to take the second sample. As a result, patients who died or progressed before week 12 were automatically excluded from this analysis. Consequently, outcome in terms of PFS and OS was better in this group in comparison with the whole population or with the patients included in the analysis for the CAF profile determined at baseline (median PFS: 4.2 months vs 3.0 months and median OS 13.5 months vs 10.5 months).

As shown in Table 3, sVEGFR2 decreased during treatment, whereas PIGF increased. The PIGF levels were below the detection threshold in 20 patients at baseline as a consequence of which the week 12/baseline ratio could not be precisely computed. Therefore, this parameter was not further analysed for PlGF.

The sVEGFR2 levels at week 12 were correlated to baseline levels (Pearson's correlation coefficient $0.537(P=0.0015)$ ). For all patients, week 12 levels were below the initial level. The PIGF levels 

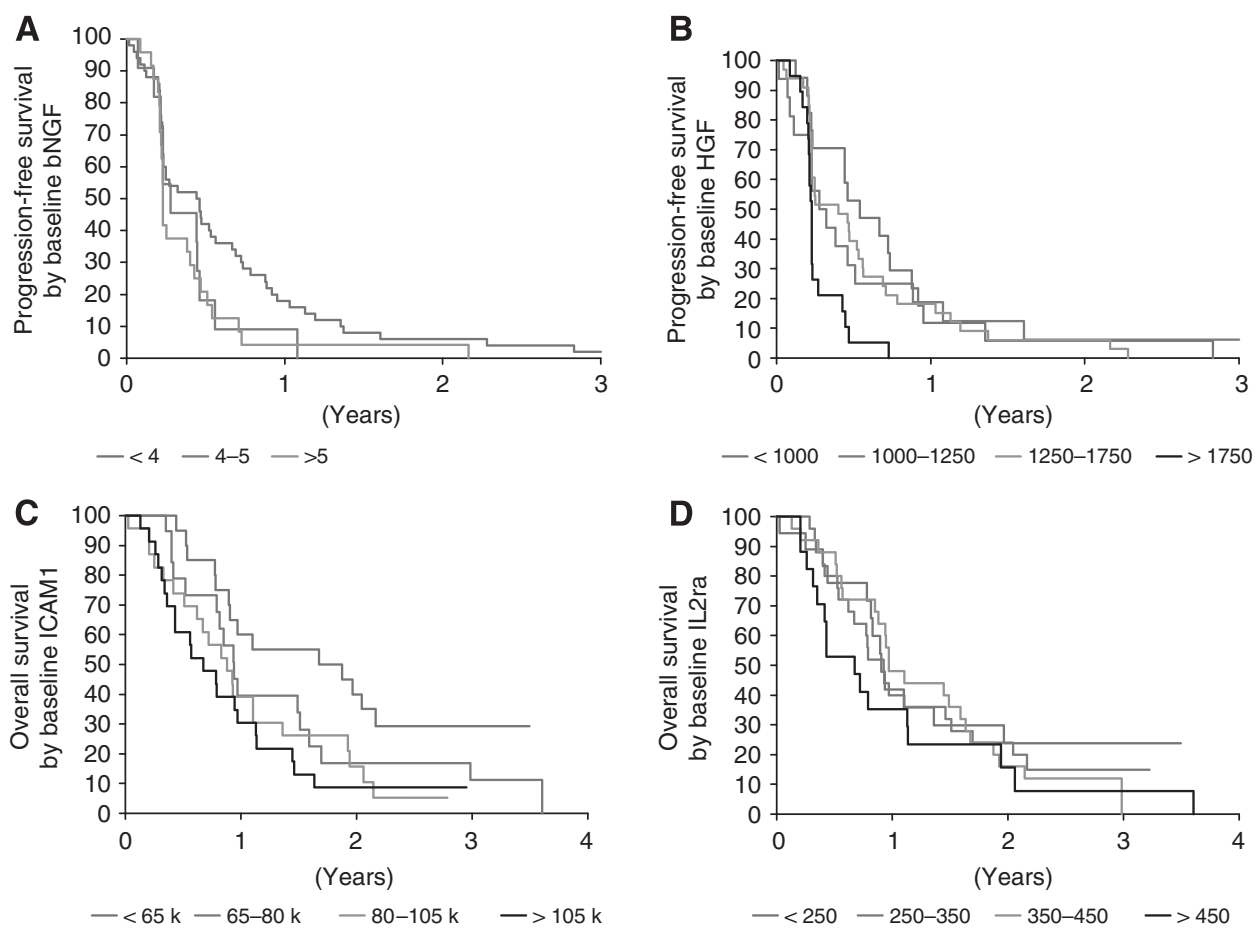

Figure I Progression-free survival by baseline bNGF $(\mathbf{A})$ and baseline HGF $(\mathbf{B})$, together with overall survival by baseline ICAM-I (C) and IL2ra (D). For this analysis, marker levels have been categorised, according to 'rounded' quartiles of their distribution. Results therefore slightly differ from those of the Cox regression analysis.

Table 3 PIGF and sVEGFR2 levels at baseline and week 12 of treatment (all concentrations are in $\mathrm{pg} \mathrm{ml}^{-1}$ )

\begin{tabular}{lcc}
\hline & Serum level of sVEGFR2 & Serum level of PIGF \\
\hline Number of observations & 32 & 31 \\
Serum level at baseline & & \\
$\quad$ Median & $10605^{\mathrm{a}}$ & $7.0^{\mathrm{a}}$ \\
Range & $6901-14898$ & $7.0-360^{\mathrm{a}}$ \\
Mean & 10690 & 23 \\
Standard deviation & 1741 & 64 \\
Serum level at week 12 & & \\
Median & 6973 & 49 \\
Range & $3193-10520$ & $7.0-356^{\mathrm{a}}$ \\
Mean & 6821 & 122 \\
s.d. & 1973 & 120 \\
& & \\
Ratio (week 12/baseline) & 0.70 & n.d. \\
Median & $0.37-0.94$ & n.d. \\
Range & 0.64 & n.d. \\
Mean & 0.16 & n.d. \\
s.d. & & \\
\hline
\end{tabular}

Abbreviations: n.d. $=$ not determined; PIGF= placental-derived growth factor sVEGFR = soluble vascular endothelial growth factor receptor. Ratio week I2/baseline was not determined for PIGF, as 20 patients had PIGF levels below threshold at baseline. ${ }^{a}$ Below detection threshold.

at week 12 were weakly correlated to baseline levels, but this may be due to the large proportion of values below the detection threshold at baseline. There was no correlation between the baseline values of sVEGFR2 and PIGF; however, the week 12 levels of the two markers showed a strong, inverse correlation (Pearson's correlation coefficient $-0.81156(P<0.001$; Figure 2$)$. There was no apparent correlation of the initial marker levels with other

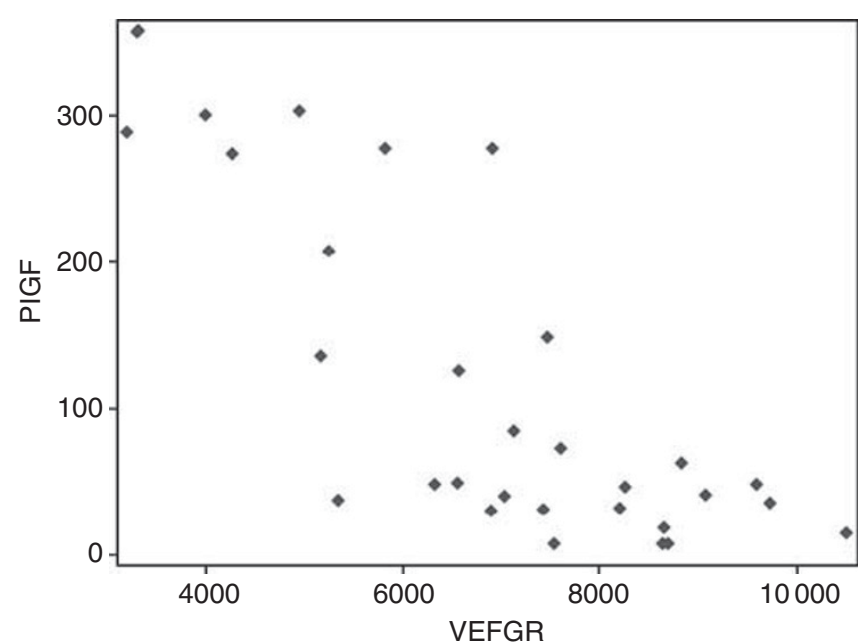

Figure 2 Scatterplot of PIGF and sVEGFR2 levels at week 12.

baseline characteristics such as age, gender, performance score, site of primary tumour, time since diagnosis, histology of the primary tumour, or tumour grade (data not shown).

To assess the correlation of these markers with hypertension, both the Pearson's (parametric) and Spearman's (non parametric) correlation coefficient were computed. Both analyses showed that hypertension was not correlated to the baseline marker levels, but was highly correlated to the marker levels at week 12 . Higher grades of hypertension were observed for patients with low sVEGFR2 levels at week 12 (Pearson's correlation coefficient $-0.57282 \quad(P=0.0006)$; Spearman's correlation coefficient $-0.57828(P=0.0005))$ and high PIGF levels at week 12 (Pearson's 
correlation coefficient $0.61287(P=0.0002)$; Spearman's correlation coefficient $0.53414(P=0.0020))$. For sVEGFR2, the week $12 /$ baseline ratio was also correlated, but weaker (Pearson's correlation coefficient $-0.50304(P=0.0033)$; Spearman's correlation coefficient $-0.49536(P=0.0039)$. Figure 3 illustrates the correlation between marker levels at week 12 and the grade of hypertension; for this graph, the marker levels were dichotomised according to the median. As sVEGFR2 and PlGF levels at week 12 were strongly correlated, also the combinations were explored. Those patients with a PlGF above and a sVEGFR2 below the respective medians had the highest grades of hypertension.

With respect to the other VEGFR-TKI-specific toxicity taken as primary end point in this study, hypopigmentation, no correlation with sVEGFR2 or PlGF levels was found. Also none of the other toxicities (fatigue, diarrhoea, and nausea) were correlated with these two markers at both time points, except for TSH elevation. Low sVEGR2 (Pearson's correlation coefficient $-0.50632 \quad(P=0.007)$; Spearman's correlation coefficient $-0.50216(P=0.0076))$ and high PlGF levels (Pearson's correla- tion coefficient $0.47833 \quad(P=0.0134)$; Spearman's correlation coefficient $0.54415(P=0.0041))$ were associated with higher TSH elevations, indicating compromised thyroid function.

As a secondary end point, the correlations of sVEGFR2 and PlGF with $\mathrm{PFS}_{12 \mathrm{wks}}$ were assessed. Baseline levels were non-predictive, but week 12 levels of both markers were significantly correlated with success rate. High sVEGFR2 and low PlGF were associated with a more favourable outcome in terms of $\mathrm{PFS}_{12 \mathrm{wks}}$ (OR 0.636; 95\% CI $0.413-0.977 \quad(P=0.039)$ ) and (OR $1.081 ; 95 \% \quad$ CI 1.007-1.160 $(P=0.0318)$, respectively) (Figure 4A). In contrast to PFS, for which no correlation could be found, there was also a correlation between marker levels at week 12 and OS. For high sVEGFR2 at week 12, there was a trend for prolonged OS (sVEGFR2: HR 0.817; 95\% CI 0.644-1.037 $(P=0.0966)$, whereas there was a statistically significant association between low PlGF levels at week 12 and longer OS (PlGF: HR 1.061; 95\% CI 1.025-1.099 $(P=0.0009)$, respectively; Figure 4B and C). Also in a multivariate model with these two factors, the association between PIGF levels at week 12 and OS was statistically significant.

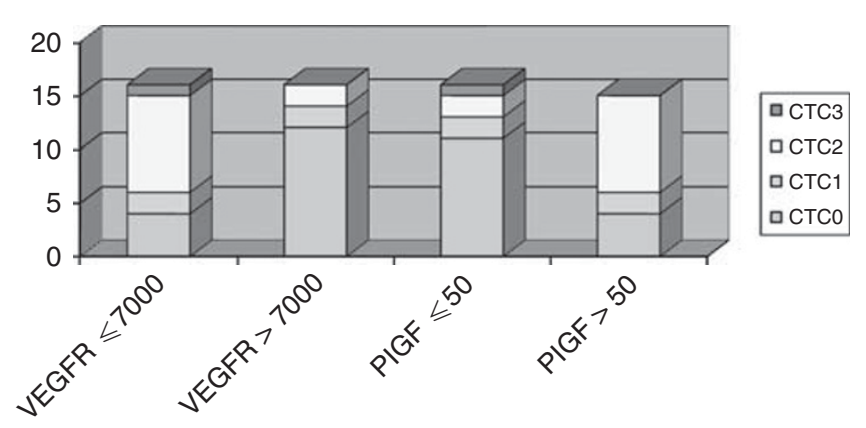

Figure 3 Associations with grade hypertension with sVEGR2 and PIGF levels at week 12.

\section{DISCUSSION}

We have previously reported on the phase II study of clinical efficacy of pazopanib in pretreated non-adipocytic STS (Sleijfer et al, 2009). This preliminary evidence has been subsequently evaluated with a placebo-controlled trial on pazopanib in pretreated non-adipocytic STS and recruitment has now been completed. The early data has shown a statistically significant three-fold prolongation in PFS with pazopanib compared with placebo (Van der Graaf et al, 2011). In the earlier study, we explored whether or not we could identify advanced STS patients likely to benefit from pazopanib on the basis of CAFs determined before treatment start. Furthermore, we did an exploratory analysis whether changes in sVEGFR2 and PlGF were associated with pazopanib-specific adverse events and anti-tumour activity.
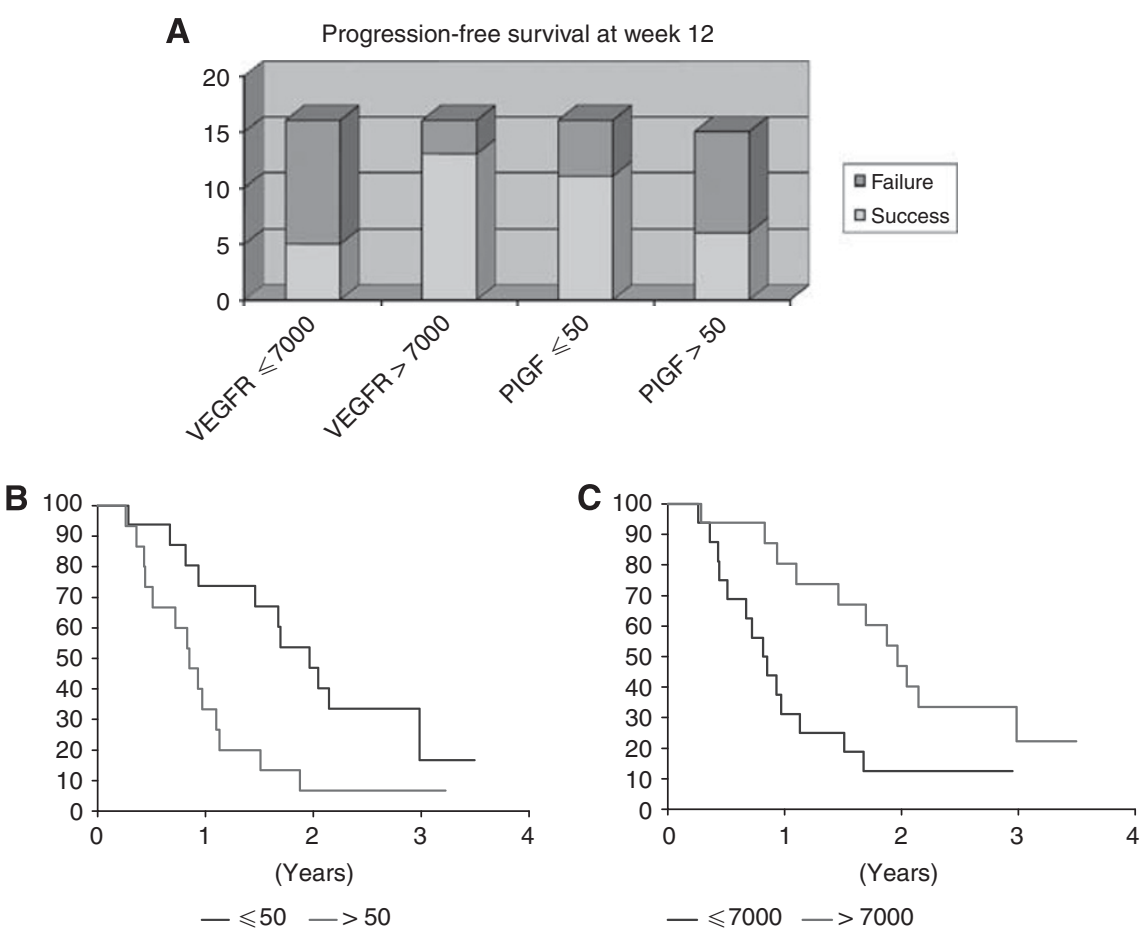

Figure 4 Association of sVEGFR2 and PIGF levels at week 12, with success rate at week 12 (A), OS by PIGF levels at week 12 (B), and by sVEGFR2 levels at week $12(\mathbf{C})$. 
Of the 23 different CAFs determined at baseline, it appeared that low IL12p40 and MPC3 levels were associated with better PFS ${ }_{12 w k s}$, low bNGF and HGF with a better PFS, and low ICAM-1 and IL2ra with prolonged OS. As inhibition of angiogenesis is thought to be the main mechanism by which pazopanib and other VEGFR-TKIs exert their anti-tumour activity, baseline factors associated with outcome may be similar across different tumour types and VEGFR-TKIs. In line with our finding of low baseline HGF levels being associated with prolonged PFS, greater tumour shrinkage and a longer PFS was observed in pazopanib-treated renal cell carcinoma (RCC) with low baseline HGF (Tran et al, 2010). In contrast, Nikolinakos et al (2010) found an association between greater tumour shrinkage and high baseline HGF in pazopanibtreated non-small cell lung carcinoma (NSCLC) patients . Also our finding that a low IL2ra was associated with a longer OS is in contrast to their findings, wherein a high IL2ra level was associated with greater tumour shrinkage in the same patient cohort (Nikolinakos et al, 2010). Other baseline factors associated with outcome with pazopanib in RCC and NSCLC were either not incorporated in our study (IL-6, IL-8, IL-12, IL-16, E-selectin, IP-10) or not associated with outcome in STS (SDF-1 $\alpha$, IL2ra, IL-3, IFN $\alpha 2$, TRAIL, M-CSF, and PlGF; Nikolinakos et al, 2010; Tran et al, 2010). With respect to VEGFR-TKIs other than pazopanib, levels of numerous serum factors, in particular VEGF and sVEGFR2, have been explored for their association with outcome in various tumour types (Murukesh et al, 2010). In some of these studies, low baseline levels were associated with a favourable outcome parameter (Kiura et al, 2008; Rini et al, 2008; Hanrahan et al, 2009b), but as was seen in our study, no such associations could be revealed in many other studies (Murukesh et al, 2010). Altogether, given the fact that none of the factors evaluated was associated with more than one efficacy parameter in our study, the high false-discovery rate ranging between $20-50 \%$, and the inconsistency associations seen for other tumour types and other VEGFR-TKIs, it can be suspected that the associations in our study are false-positive ones. Nevertheless, given the potentially important clinical relevance, the identified associations deserve to be studied further and will therefore be investigated in the recently completed placebo-controlled phase III study on pazopanib in non-adipocytic STS patients.

In addition to baseline CAFs, we explored whether absolute sVEFR2 and PlGF levels at week 12 after treatment start and/or changes compared with baseline are associated with pazopanibspecific-toxicities and with outcome to pazopanib. Previously, several VEGFR-TKI such as pazopanib, sunitinib, cediranib, vandetanib, and motesanib have consistently been shown to decrease sVEGFRs levels and to increase VEGF and PlGF (Drevs et al, 2005; Rini et al, 2008; Hanrahan et al, 2009a; Bass et al, 2010; Murukesh et al, 2010; Nikolanikos et al, 2010) and can therefore be considered a class effect. There is preclinical evidence in sunitinibtreated tumour-bearing mice for an association between these changes in VEGF and P1GF levels and tumour response (Ebos et al, 2007). Therefore, these markers have been proposed as pharmacodynamic markers for the extent of VEGFR inhibition. As mentioned, VEGFR inhibition induces increases in both VEGF and PlGF. As increases in PlGF are more pronounced than those observed for VEGF (Rini et al, 2008; Nikolanikos et al, 2010), we chose for the latter in this study. As expected, pazopanib in our study decreased sVEGR2 and increased PIGF. Consistent with the hypothesis that these alterations reflect the extent of inhibition of VEGFR activity, they were associated with the occurrence of hypertension and TSH elevation, which are both side effects considered specific for VEGFR-TKIs. This further supports the hypothesis that changes in these markers may act as pharmacodynamic markers for VEGFR inhibition. In contrast to our findings, associations of changes in VEGF and sVEGFR2 with hypertension were not observed in sunitinib-treated RCC patients (Rini et al, 2008). We could not find any associations with the occurrence of fatigue and diarrhoea, but it is recognised that there may be multifactorial causes for these events in cancer patients, including the disease itself and concomitant medications.

Whether or not changes in these factors are related to efficacy of VEGFR-TKIs is of major interest. In a preclinical model, low sVEGFR2 and high VEGF and PlGF levels, indicative for VEGFR inhibition, were associated with VEGFR-TKI-mediated antitumour activity (Ebos et al, 2007). In pazopanib-treated NSCLC patients, an association between a decrease in sVEGFR2 and tumour shrinkage was found, whereas changes in PlGF or VEGF were not. Also in a phase I study with AMG706 in advanced cancer patients, high PlGF and low sVEGFR2 levels were correlated with greater tumour shrinkage (Rosen et al, 2007). In addition, in a phase II study on patients with advanced thyroid cancer treated with motesanib, the response rate was $30 \%$ in patients having a 4.7-fold increase in PlGF at 1 week after treatment start compared with a $3 \%$ response rate in those patients with a less profound increase. Also patients with the strongest decrease in sVEGFR2 had a higher chance to respond favourably to motesanib (Bass et al, 2010). However, in several other studies exploring VEGFR-TKIs in various tumour types, changes in these markers were not associated with outcome (Murukesh et al, 2010). Unexpectedly, low sVEGFR2 and high PlGF levels at week 12 were not only associated with a poorer $\mathrm{PFS}_{12 \mathrm{wks}}$, the primary efficacy end point in this study, but also with a worse OS. Interestingly and consistent with our findings, an increase in VEGF at day 8 after initiation of treatment with the VEGFR-TKI vandetanib was correlated with a greater risk of progression in NSCLC patients (Hanrahan et al, 2009a). Why effects suggestive of adequate VEGFR inhibition are related to a poorer outcome to VEGFR inhibitors is unknown. Lower sVEGFR2 levels, potentially leading to higher circulating levels of free VEGF, and increased PlGF levels that occur as response to VEGFR inhibition are probably physiological mechanisms to overcome and escape the effects of VEGFR blockade. Continuous pressure of these increased levels may ultimately lead to activation of the respective receptors, VEGFR1 and VEGFR2 (Fischer et al, 2008), thereby stimulating tumour cell growth and angiogenesis, and overcoming the inhibitory effects of pazopanib. But still, this does not explain why high PlGF and low sVEGFR2 levels are associated with favourable outcomes in some studies, whereas the contrary happens in other studies, including ours. Maybe some tumour types, including STS, are more sensitive for the proangiogenic and stimulating effects of PlGF than other malignancies, thereby partially counteracting the inhibitory effects from VEGFR-TKIs in these particular tumour types. However, this is purely speculative and required further study.

Irrespective of the underlying mechanisms, if these associations can be confirmed, changes in VEGF and PlGF form attractive markers to determine whether or not a patient benefits from VEGFR-TKI, and already at an early point during treatment. This will hold true in particular when such associations exist as early as day 8 of treatment as was found in vandetanib-treated NSCLC patients (Hanrahan et al, 2009a). To validate these potentially important associations, the same markers will be determined at several time points in various EORTC studies on pazopanib in diverse tumour types, including the pazopanib phase III trial in non-adipocytic STS.

In conclusion, several baseline CAFs were related to efficacy of pazopanib in advanced STS patients. However, these exploratory results should be interpreted with caution given high falsediscovery rates and inconsistency between the diverse efficacy parameters. Indicative of VEGFR inhibition, pazopanib decreases sVEGFR2 and increases PlGF levels, and changes of both factors are associated with the pazopanib-specific toxicities hypertension and TSH elevations. The associations of these changes with poorer efficacy in terms of $\mathrm{PFS}_{12 \mathrm{wks}}$ and OS were unexpected but may be 
of great clinical value. Findings of this study will be validated in the recently completed phase III study of pazopanib in non-adipocytic STS. If confirmed, these factors may be used as early markers for response to pazopanib enabling further individualisation of STS treatment.

\section{REFERENCES}

Bass MB, Sherman SI, Schlumberger MJ, Davis MT, Kivman L, Khoo HM, Notari KH, Peach M, Hei YJ, Patterson SD (2010) Biomarkers as predictors of response to treatment with motesanib in patients with progressive advanced thyroid cancer. J Clin Endocrinol Metab 95: 5018-5027

Demetri GD, Chawla SP, von Mehren M, Ritch P, Baker LH, Blay JY, Hande KR, Keohan ML, Samuels BL, Schuetze S, Lebedinsky C, Elsayed YA, Izquierdo MA, Gomez J, Park YC, Le Cesne A (2009) Efficacy and safety of trabectidin in patients with advanced or metastatic liposarcoma or leiomyosarcoma after failure of prior anthracyclines and ifosfamide: results of a randomized phase II study of two different schedules. J Clin Oncol 27: 4188-4196

Drevs J, Zirrgiebel U, Schmidt-Gersbach CI, Mross K, Medinger M, Lee L, Pinheiro J, Wood J, Thomas AL, Unger C, Henry A, Steward WP, Laurent D, Lebwohl D, Dugan M, Marme D (2005) Soluble markers for the assessment of biological activity with PTK787/ZK222584 (PTK/ZK), a vascular endothelial growth factor receptor (VEGFR) tyrosine kinase inhibitor in patients with advanced colorectal cancer from two phase I trials. Ann Oncol 16: 558-565

Ebos JM, Lee CR, Christensen JG, Mutsaerts AJ, Kerbel RS (2007) Multiple circulating proangiogenic factors induced by sunitinib malate are tumorindependent and correlate with antitumor efficacy. Proc Natl Acad Sci USA 104: 17069-17074

Fata F, O'Reilly E, Ilson D, Pfister D, Leffel D, Kelsen DP, Schwartz GK, Casper ES (1999) Paclitaxel in the treatment of patients with angiosarcoma of the scalp or face. Cancer 86: 2034-2037

Fischer C, Mazzone M, Jonckx B, Carmeliet P (2008) FLT1 and its ligands VEGFB and PlGF: drug targets for anti-angiogenic therapy? Nat Rev Cancer 8: 942-956

Hamberg P, Verweij J, Sleijfer S (2010) (Pre)clinical pharmacology and activity of pazopanib, a novel multikinase angiogenesis inhibitor. Oncologist 15: 539-547

Hanrahan EO, Lin HY, Kim ES, Yan S, Du DZ, McKee KS, Tran HT, Lee JJ, Ryan AJ, Langmuir P, Johnson BE, Heymach JV (2009a) Distinct patterns of cytokine and angiogenic factor modulation and markers of benefit for vandetanib and/or chemotherapy in patients with non-small cell lung cancer. J Clin Oncol 28: 193-201

Hanrahan EO, Ryan AJ, Mann H, Kennedy SJ, Langmuir P, Natale RB, Herbst RS, Johnson BE, Heymach JV (2009b) Baseline vascular endothelial growth factor concentration as a potential predictive marker of benefit from vandetanib in non-small lung cancer. Clin Cancer Res 15: $3600-3609$

Kiura K, Nakagawa K, Shinkai T, Eguchi K, Ohe Y, Yamamoto N, Tsuboi M, Yokota S, Seto T, Jiang H, Nishio K, Saijo N, Fukuoka M (2008) A randomized, double-blind, phase IIa dose-finding study of vandetanib (ZD6474) in Japanese patients with non-small cell lung cancer. J Thorac Oncol 3: 386-393

\section{ACKNOWLEDGEMENTS}

We gratefully acknowledge Sabine van Steenbergen and Brigitte van Krimpen for their technical assistance. This study was financially supported by GlaxoSmithKline.
Murukesh N, Dive C, Jayson GC (2010) Biomarkers of angiogenesis and their role in the development of VEGF inhibitors. Br J Cancer 102: 8-18 Nikolanikos PG, Altorki N, Yankelevitz D, Tran HT, Yan S, Rajagopalan D, Bordogna W, Ottesen LH, Heymach JV (2010) Plasma cytokine and angiogenic factor profiling identifies markers associated with tumor shrinkage in early-stage non-small cell lung cancer patients treated with pazopanib. Cancer Res 70: 2171-2179

Rini BI, Michaelson MD, Rosenberg JE, Bukowski RM, Sosman JA, Stadler WM, Hutson TE, Margolin K, Harmon CS, DePrimo SE, Kim ST, Chen I, George DJ (2008) Antitumor activity and biomarker analysis of sunitinib in patients with bevacizumab-refractory metastatic renal cell carcinoma. J Clin Oncol 26: 3743-3748

Rosen LS, Kurzrock R, Mulay M, Van Vugt A, Purdom M, Ng C, Silverman J, Koutsoukos A, Sun YN, Bass MB, Xu RY, Polverino A, Wiezorek JS, Chang DD, Benjamin R, Herbst RS (2007) Safety, pharmacokinetics, and efficacy of AMG 706, an oral multikinase inhibitor, in patients with advanced solid tumors. J Clin Oncol 25: 2369-2376

Sleijfer S, Ray-Coquard I, Papai Z, Le Cesne A, Scurr M, Schöffski P, Collin F, Pandite L, Marreaud S, De Brauwer A, van Glabbeke M, Verweij J, Blay JY (2009) Pazopanib, a multikinase angiogenesis inhibitor, in patients with relapsed or refractory advanced soft tissue sarcoma: a phase II study from the European Organisation for Research and Treatment of CancerSoft Tissue and Bone Sarcoma group (EORTC 62043). J Clin Oncol 27: 3126-3132

Sleijfer S, Seynaeve C, Verweij J (2005) Using single-agent therapy in adult patients with advanced soft tissue sarcoma can still be considered standard care. Oncologist 10: 833-841

Tascilar M, Loos WJ, Seynaeve C, Verweij J, Sleijfer S (2007) The pharmacologic basis of ifosfamide use in adult patients with advanced soft tissue sarcomas. Oncologist 12: 1351-1360

Tran HT, Liu Y, Lin Y, Martin A-M, Baker KL, Fritsche HA, Zurita A, Pandite LN, Heymach JV (2010) Use of multiplatform analysis of plasma cytokines and angiogenic factors (CAFs) to identify baseline CAFs associated with pazopanib response and tumor burden in renal cell carcinoma (RCC) patients. J Clin Oncol 28 (suppl 15s): abstr 4522

Van der Graaf WT, Blay JY, Chawla SP, Kim D, Bui Nguyen B, Casali PG, Schöffski P, Aglietta M, Staddon AP, Beppu Y, Le Cesne A, Gelderblom H, Judson IR, Araki N, Ouali M, Marreaud S, Hodge R, Dewji M, Dei Tos AP, Hohenberger P (2011) PALETTE: a randomized, double-blind, phase III trial of pazopanib versus placebo in patients (pts) with soft-tissue sarcoma (STS) whose disease has progressed during or following prior chemotherapy-an EORTC STBSG Global Network Study (EORTC 62072). J Clin Oncol 29 (suppl): abstr LBA10002

Verweij J, Casali PG, Zalcberg J, LeCesne A, Reichardt P, Blay JY, Issels R, van Oosterom A, Hogendoorn PC, Van Glabbeke M, Bertulli R, Judson I (2004) Progression-free survival in gastrointestinal stromal tumours with high-dose imatinib: a randomised trial. Lancet 364: 1127-1134

This work is published under the standard license to publish agreement. After 12 months the work will become freely available and the license terms will switch to a Creative Commons Attribution-NonCommercial-Share Alike 3.0 Unported License. 